\title{
Tumor-Infiltrating Podoplanin+ Fibroblasts Predict Worse Outcome in Solid Tumors
}

\author{
Guoming $\mathrm{Hu}^{\mathrm{a}}$ Shimin Wang ${ }^{\mathrm{b}}$ Feng $\mathrm{Xu}^{\mathrm{a}}$ Qiannan Ding ${ }^{\mathrm{c}}$ Wei Chen ${ }^{\mathrm{a}}$ \\ Kefang Zhong ${ }^{\mathrm{a}}$ Liming Huang $^{\mathrm{a}}$ Qi Xu ${ }^{\mathrm{d}}$
}

${ }^{a}$ Department of General Surgery (Breast and Thyroid Surgery), Shaoxing People's Hospital (Shaoxing Hospital, Zhejiang University School of Medicine), Zhejiang, bDepartment of Nephrology, Shaoxing People's Hospital (Shaoxing Hospital, Zhejiang University School of Medicine), Zhejiang, 'Medical Research Center, Shaoxing People's Hospital (Shaoxing Hospital, Zhejiang University School of Medicine), Zhejiang, dDepartment of Surgical Oncology, Shaoxing Second Hospital, Zhejiang, China

\section{Key Words}

Podoplanin ${ }^{+}$fibroblasts $\cdot$ Tumor microenvironment $\cdot$ Worse outcome $\cdot$ Solid tumor $\cdot$ Metaanalysis

\begin{abstract}
Background/Aims: Tumor-infiltrating fibroblasts are a heterogeneous population, and different subpopulations play differential roles in tumor microenvironment. However, the prognostic role of podoplanin ${ }^{+}$fibroblasts in human solid tumors still remains controversial. Therefore, we performed the meta-analysis to better understand the role of this subpopulation in prognosis prediction for patients with solid tumor. Methods: We searched PubMed and EBSCO to identify the studies evaluating the association of intratumoral podoplanin ${ }^{+}$fibroblast density detected by immunohistochemical method and overall survival (OS) and/or diseasefree survival (DFS) in patients with solid tumor, then computed extracted data into hazard ratios for OS, DFS and clinicopathological features with STATA 12.0. Results: A total of 4883 patients from 29 published studies were incorporated into this meta-analysis. We found that podoplanin+ fibroblast infiltration significantly decreased OS and DFS in all types of solid tumors. In stratified analyses, podoplanin+ fibroblast infiltration was significantly associated with worse OS in cholangiocarcinoma, breast, lung and pancreatic cancer. And these cells were inversely associated with DFS in breast, lung and pancreatic cancer. In addition, high density of these cells significantly correlated with unfavorable clinicopathological features such as lymph node metastasis, TNM stage, lymphatic and vascular invasion of solid tumor. Conclusion: Podoplanin ${ }^{+}$fibroblast infiltration leads to worse clinical outcome in solid tumors, implicating that it is a valuable prognostic biomarker and targeting it may have a potential for effective treatment.




\section{Cellular Physiology Cell Physiol Biochem 2018;51:1041-1050 and Biochemistry \begin{tabular}{l|l} 
DOI: 10.1159/000495484 & (c) 2018 The Author(s). Published by S. Karger AG, Basel \\
www.karger.com/cpb
\end{tabular}}

Hu et al.: Prognostic Role of Tumor-Infiltrating Podoplanin ${ }^{+}$Fibroblasts

\section{Introduction}

Accumulating evidence has demonstrated that tumor-infiltrating fibroblasts were significantly associated with survival of solid tumors. However, they are a heterogeneous population, thus, it is important to distinguish among different subpopulations of them as they may play differential roles in tumor microenvironment (TME) [1]. Podoplanin ${ }^{+}$ fibroblasts, a new subpopulation of fibroblasts identified recently, have been demonstrated to play specific and significant roles in a number of human solid tumors.

Podoplanin, a well-conserved, mucin-type transmembrane protein, has exerted a variety of functions including regulation of organ development and cell motility [2]. Recent studies have indicated that podoplanin was upregulated in fibroblasts in the tumor stroma [3]. Podoplanin ${ }^{+}$fibroblasts are often among the early immune cells recruited to tumor sites in response to the stimuli and increase in the TME. In the past decades, multitudinous studies have associated tumor-infiltrating podoplanin ${ }^{+}$fibroblasts and prognosis in patients with solid tumor, but their results were controversial [4]. Thus, it needs in-depth assessment. Furthermore, the potential of these cells as a prognostic biomarker and targeted immunotherapy is essential to be explored.

We performed this meta-analysis to clarify the association between the infiltration of podoplanin ${ }^{+}$fibroblasts and outcomes such as overall survival (OS) and disease-free survival (DFS) in breast cancer patients.

\section{Materials and Methods}

\section{Search strategy}

PubMed and EBSCO were searched for studies to assess the density of podoplanin ${ }^{+}$fibroblasts and survival in patients with solid tumor from 1980 to March 31th 2018. The keywords adopted for search were (podoplanin [Title/Abstract] OR fibroblasts [Title/Abstract]) AND (neoplasms [Title/Abstract] OR tumor [Title/Abstract] OR cancer [Title/Abstract] OR carcinoma [Title/Abstract]) AND (prognosis [Title/ Abstract] OR survival [Title/Abstract]).

\section{Inclusion and exclusion criteria}

In this meta-analysis, the inclusion criteria were: studies must have (1) been published as original articles; (2) investigated human subjects with solid tumor; (3) measured podoplanin ${ }^{+}$fibroblasts in primary tumor specimens with immunohistochemistry (IHC); (4) provided hazard ratios (HRs) with 95\% confidence interval (CI), or Kaplan - Meier curves of high and low podoplanin+ fibroblast density with OS, and/or DFS; (5) been published in English.

The exclusion criteria were: studies (1) were not published as research articles or full texts including commentary, case report, letters to editors and conference abstracts; (2) didn't provide sufficient data to estimate HRs; (3) detected fibroblasts not with marker 'podoplanin', or in metastatic tissues.

\section{Endpoints}

In this study, we recorded OS and DFS as the primary and second endpoint respectively.

\section{Data extraction}

Two authors (GM.H. and W.C.) independently reviewed and extracted data such as first author's name, publication year, number of patients, median age, time of follow-up, method applied to quantify podoplanin ${ }^{+}$ fibroblasts, and cut-off value to determine high density of these cells. OS, DFS and clinicopathological data including Tumor, Lymph Node, Metastasis (TNM) stage, lymphatic invasion etc were extracted from the text, tables, or Kaplan - Meier curves. 


\section{Cellular Physiology Cell Physiol Biochem 2018;51:1041-1050 and Biochemistry Published $\begin{aligned} & \text { DOI: } 10.1159 / 000495484 \\ & \text { (c) } 2018 \text { The Author(s). Published by S. Karger AG, Basel } \\ & \text { www.karger.com/cpb }\end{aligned}$}

Quality assessment

Two independent authors evaluated the quality of included cohort studies with Newcastle-Ottawa Scale (NOS) [5] and achieved consensus for each item under the help of third author. A total score of 6 or more points was considered high quality.

\section{Statistical Analysis}

We combined extracted data into meta-analyses with STATA 12.0 analysis software (Stata Corporation, College Station, TX, USA). Statistical heterogeneity was evaluated by the chi-squared based Q-test or $I^{2}$ method [6]. Data were combined according to the random-effect model in the presence of heterogeneity [7] otherwise, the fixed-effect model was applied [8]. Sensitivity analysis, Begg's funnel plot and Egger's test [9] were adopted to investigate the influence of each study on the pooled result and potential publication bias respectively. All $P$ values were two-sided and less than 0.05 are considered statistically significant.

\section{Results}

\section{Search results and description of studies}

10694 records were retrieved and the results were exhibited in Fig. 1. We ultimately identified 29 studies containing 4883 patients with solid tumor for the assessment of intratumoral podoplanin ${ }^{+}$fibroblasts [10-38] and then evaluated all these studies with the Newcastle-Ottawa Scale (NOS). Characteristics of included studies that were in accordance with the inclusion criteria and suitable for data consolidation were shown in Table 1.

\section{Meta-analyses}

Overall survival (OS). The meta-analysis showed that podoplanin ${ }^{+}$fibroblast infiltration was significantly associated with decreased OS (HR $=1.77,95 \%$ CI 1.42 to $2.20, P<0.001)$ in patients with solid tumor (Fig. 2). In stratified analyses according to cancer types, as shown in Fig. 3, pooled results indicated that elevated density of podoplanin ${ }^{+}$fibroblasts was significantly associated with worse OS in breast cancer (BC) $(\mathrm{HR}=2.46,95 \%$ CI 1.73 to $3.50, P<0.001$ ), with no heterogeneity detected $\left(I^{2}=0 \%\right.$, $P=0.840$ ); Similar results were observed between increased density of these cells and OS in lung cancer $(\mathrm{LC})(\mathrm{HR}=1.68$, $95 \%$ CI 1.18 to $2.41, P=0.004)$, pancreatic cancer $(\mathrm{PC})(\mathrm{HR}=$ $2.20,95 \%$ CI 1.40 to $3.46, P=$ 0.001 ), and cholangiocarcinoma (CC) $(\mathrm{HR}=1.92,95 \% \mathrm{CI} 1.11$ to 3.32, $P=0.019$ ). However, there was no significant association between podoplanin ${ }^{+}$fibroblast infiltration and OS in esophageal carcinoma (EC) ( $\mathrm{HR}=1.58,95 \%$ CI 0.89 to $2.81, P=0.116$ ).

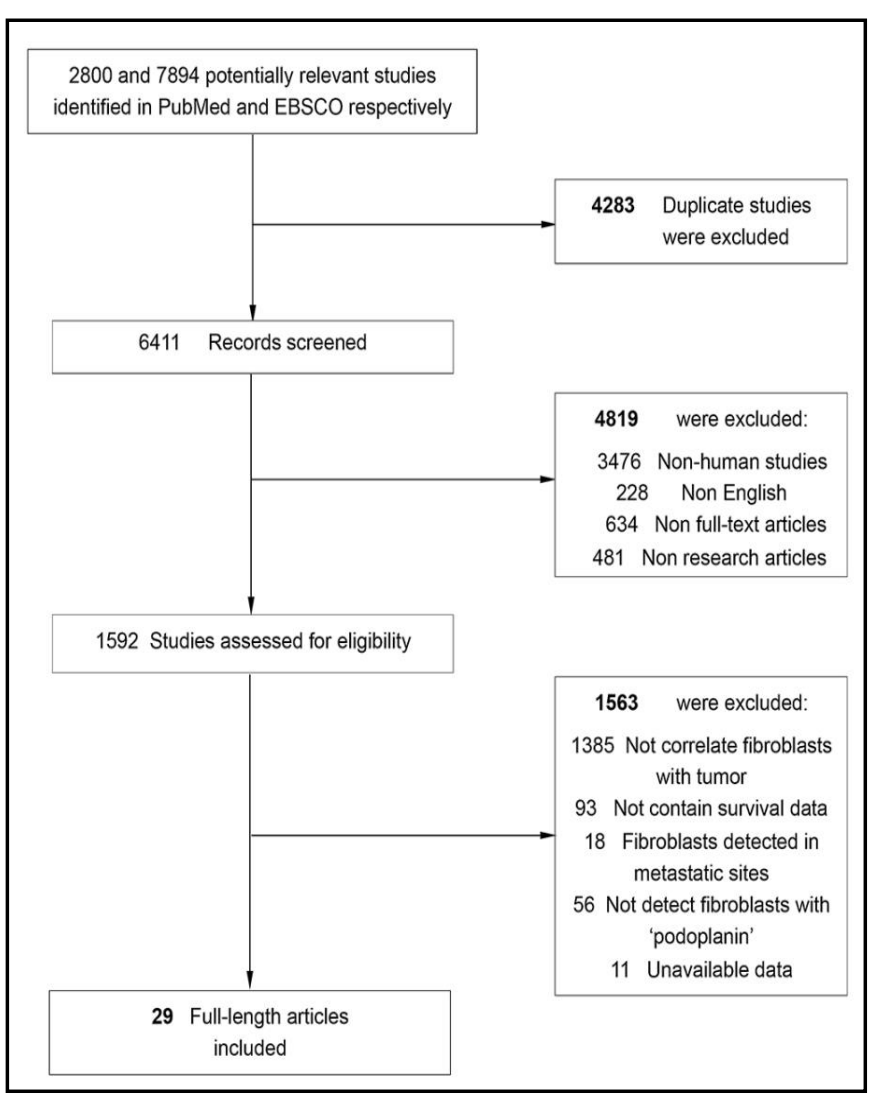

Fig. 1. Flow chart diagram of study selection. 
Table 1. Main characteristics of the included studies

\begin{tabular}{|c|c|c|c|c|c|c|c|c|c|}
\hline Study & Year & Tumor type & No. of Patients & $\mathrm{M} / \mathrm{F}$ & Cut-offs & podoplanin+ fibroblasts: $\mathrm{H} / \mathrm{L}$ & Tumor stage & Survival & NOS \\
\hline Cai, D.Y. etal [10] & 2017 & $\mathrm{BC}$ & 164 & $0 / 164$ & $\geq 10 \%$ of the stroma & $72 / 92$ & I - III & DFS & 7 \\
\hline Park, C.K. etal [11] & 2016 & $\mathrm{BC}$ & 524 & $0 / 524$ & $\geq 10 \%$ of the stroma & $101 / 423$ & I - II & OS, DFS & 8 \\
\hline Park, S.Y. etal [12] & 2015 & $\mathrm{BC}$ & 275 & $0 / 275$ & $\geq 10 \%$ of the stroma & $40 / 235$ & I - III & os & 8 \\
\hline Pula, B. etal [13] & 2013 & $\mathrm{BC}$ & 104 & $0 / 104$ & immunoreaction score $>3$ & $74 / 30$ & I - III & os & 7 \\
\hline Schoppmann, S.F. etal [14] & 2012 & $\mathrm{BC}$ & 367 & $0 / 367$ & $\geq 10 \%$ of the stroma & $33 / 334$ & I - III & OS, DFS & 8 \\
\hline Pula, B. etal [15] & 2011 & $\mathrm{BC}$ & 117 & $0 / 117$ & $\geq$ grade 1 & $96 / 21$ & $I-I V$ & OS, DFS & 7 \\
\hline Nakasone, S. etal [16] & 2018 & LAC & 97 & $51 / 46$ & $\geq 10 \%$ of spindle cells & $40 / 57$ & I - II & OS, DFS & 6 \\
\hline Kubouchi, Y. etal [17] & 2018 & Stage IA LAC & 158 & $76 / 82$ & $\geq 10 \%$ of spindle cells & $41 / 117$ & I A - I B & OS, DFS & 7 \\
\hline Yurugi, Y. etal [18] & 2017 & LSCC & 126 & $115 / 11$ & $\geq 10 \%$ of spindle cells & $41 / 85$ & $\mathrm{I}-\mathrm{II} \mathrm{A}$ & OS, DFS & 7 \\
\hline Koriyamai, H. etal [19] & 2015 & LAC & 87 & $54 / 33$ & $\geq 50 \%$ of spindle cells & $30 / 57$ & I - IV & os & 6 \\
\hline Takahashi, A. etal [20] & 2013 & LNEC & 115 & $98 / 17$ & $\geq 50 \%$ of spindle cells & $47 / 68$ & I - IV & OS, DFS & 8 \\
\hline Ono, S. etal [21] & 2013 & Stage I LSCC & 142 & $125 / 17$ & $\geq 50 \%$ of $\mathrm{CAFs}$ & $44 / 98$ & I A - I B & OS, DFS & 7 \\
\hline Neri, S. etal [22] & 2012 & Stage III LAC & 112 & $64 / 48$ & $\geq 10 \%$ of stromal fibroblasts & $51 / 61$ & III & os & 7 \\
\hline Ito, M. etal [23] & 2012 & Stage I LAC & 304 & $139 / 165$ & $\geq 10 \%$ of spindle cells & 105/199 & I A - I B & DFS & 7 \\
\hline Hoshino, A. etal [24] & 2011 & LAC & 112 & $54 / 58$ & $\geq 10 \%$ of spindle cells & $32 / 80$ & NR & OS, DFS & 7 \\
\hline Kitano, H. etal [25] & 2010 & $\mathrm{LC}$ & 266 & $182 / 84$ & $\geq 10 \%$ of spindle cells & $92 / 174$ & I - IV & os & 6 \\
\hline Kawase, A. etal [26] & 2008 & LAC & 177 & $86 / 91$ & $\geq 10 \%$ of spindle cells & $54 / 123$ & I - IV & os & 8 \\
\hline Chuang, W.Y. etal [27] & 2014 & $\mathrm{EC}$ & 59 & $58 / 1$ & $\geq 25 \%$ of stromal fibroblasts & $31 / 28$ & I - IV & os & 6 \\
\hline $\begin{array}{l}\text { Nakashima, Y. etal [28] } \\
\text { Schoppmann, S.F. etal [29] }\end{array}$ & 2013 & $\mathrm{EC}$ & 101 & $88 / 13$ & $\geq 10 \%$ of the stroma & $58 / 43$ & NR & os & $\begin{array}{l}6 \\
7\end{array}$ \\
\hline $\begin{array}{l}\text { Schoppmann, S.F. etal [29] } \\
\text { Hirayama, K etal [30] }\end{array}$ & $\begin{array}{l}2013 \\
2018\end{array}$ & $\begin{array}{l}\text { EC } \\
\text { PC }\end{array}$ & $\begin{array}{c}200 \\
95\end{array}$ & $\begin{array}{c}138 / 62 \\
57 / 38\end{array}$ & $\begin{array}{c}\geq 10 \% \text { of the stroma } \\
\geq 11.83 \% \text { of the stroma }\end{array}$ & $\begin{array}{l}44 / 156 \\
50 / 45\end{array}$ & $\begin{array}{l}\text { NR } \\
\text { I - II }\end{array}$ & $\begin{array}{l}\text { OS, DFS } \\
\text { OS, DFS }\end{array}$ & $\begin{array}{l}7 \\
8\end{array}$ \\
\hline Shindo, K. etal [31] & 2014 & $\mathrm{PC}$ & 130 & $82 / 48$ & $\geq 50 \%$ of stromal fibroblasts & $21 / 10$ & I - IV & os & 7 \\
\hline Shindo, K. etal [32] & 2013 & $\mathrm{PC}$ & 105 & $70 / 35$ & $\geq 30 \%$ of stromal fibroblasts & $74 / 31$ & I - IV & OS, DFS & 8 \\
\hline Choi, S.Y. etal [33] & 2013 & CRC & 302 & $181 / 121$ & $\geq 30 \%$ of the stroma & $125 / 177$ & I - IV & DFS & 6 \\
\hline Yamanashi, T. etal [34] & 2009 & CRC & 120 & $76 / 44$ & $\geq 30 \%$ of the stroma & $50 / 70$ & $\mathrm{NR}$ & OS, DFS & 7 \\
\hline Obulkasim, H etal [35] & 2018 & $\mathrm{CC}$ & 42 & $25 / 17$ & NR & $20 / 22$ & I - IV & os & 7 \\
\hline Aishima, S. etal [36] & 2008 & cC & 88 & $57 / 31$ & $\mathrm{NR}$ & $38 / 50$ & $\mathrm{NR}$ & os & 6 \\
\hline Kan, S. etal [37] & 2014 & Melanoma & 55 & $21 / 34$ & $\geq 10 \%$ of the stromal cells & $25 / 30$ & I - IV & os & 7 \\
\hline Sun, W.Y. etal [38] & 2015 & TPC & 339 & $272 / 67$ & $\geq 11 \%$ of the stromal cells & $90 / 249$ & NR & OS, DFS & 6 \\
\hline
\end{tabular}

\section{Disease-free survival} (DFS)

Meta-analysis showed that podoplanin ${ }^{+}$fibroblast infiltration significantly decreased DFS (HR = 1.74, $95 \%$ CI 1.25 to $2.42, P=$ 0.001 ) in solid tumors (Fig. 4). In stratified analyses by cancer types, we found that increased density of podoplanin ${ }^{+}$ fibroblasts within tumor was significantly associated with worse DFS in BC (HR $=2.26,95 \%$ CI 1.56 to $3.28, P<0.001$ ), with little heterogeneity existing among included studies $\left(I^{2}\right.$ $=6.1 \%, P=0.362$ ). Similar results were observed between podoplanin ${ }^{+}$ fibroblast infiltration and DFS in LC (HR $=1.87,95 \%$ CI 1.07 to $3.26, P=0.027)$ and PC (HR $=1.97,95 \%$ CI 1.37 to $2.84, P=0.000)$; whereas there was no significant association between podoplanin ${ }^{+}$fibroblast infiltration and DFS in CRC (HR $=0.33,95 \%$ CI 0.06 to $1.81, P=0.203$ ) (Fig. 5).

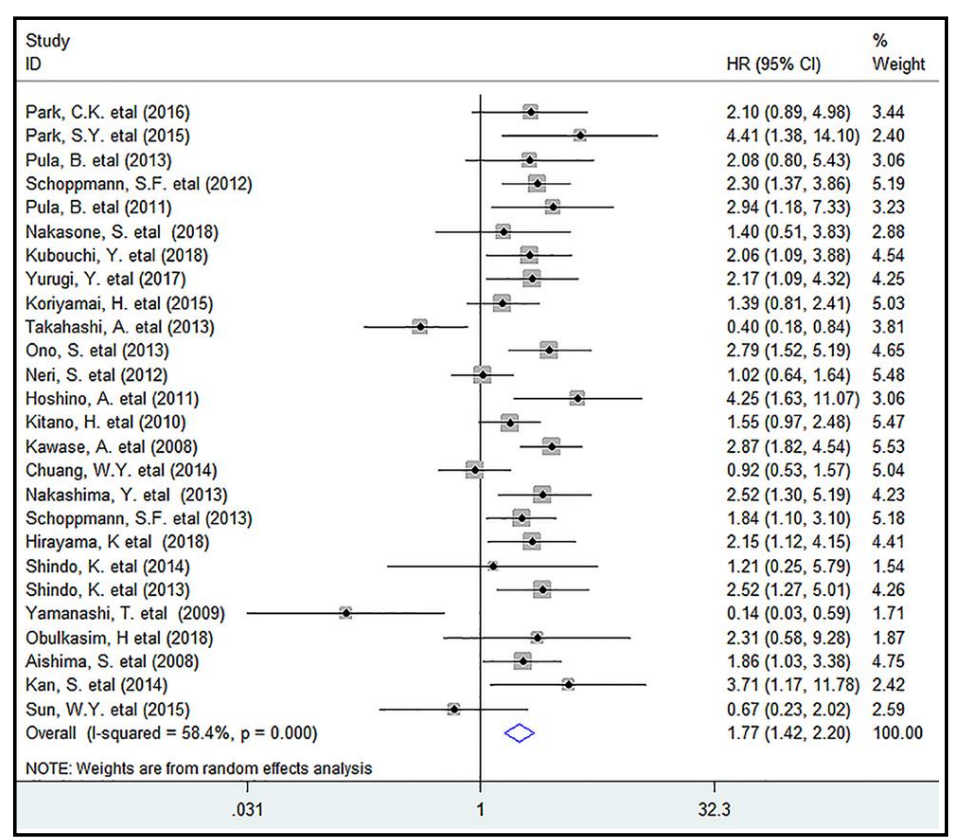

Fig. 2. Forest plot describing HR of the association between podoplanin ${ }^{+}$ fibroblast infiltration and OS in solid tumors. 
Fig. 3. Stratified analyses describing HRs of the association between podoplanin ${ }^{+}$ fibroblast infiltration and OS.

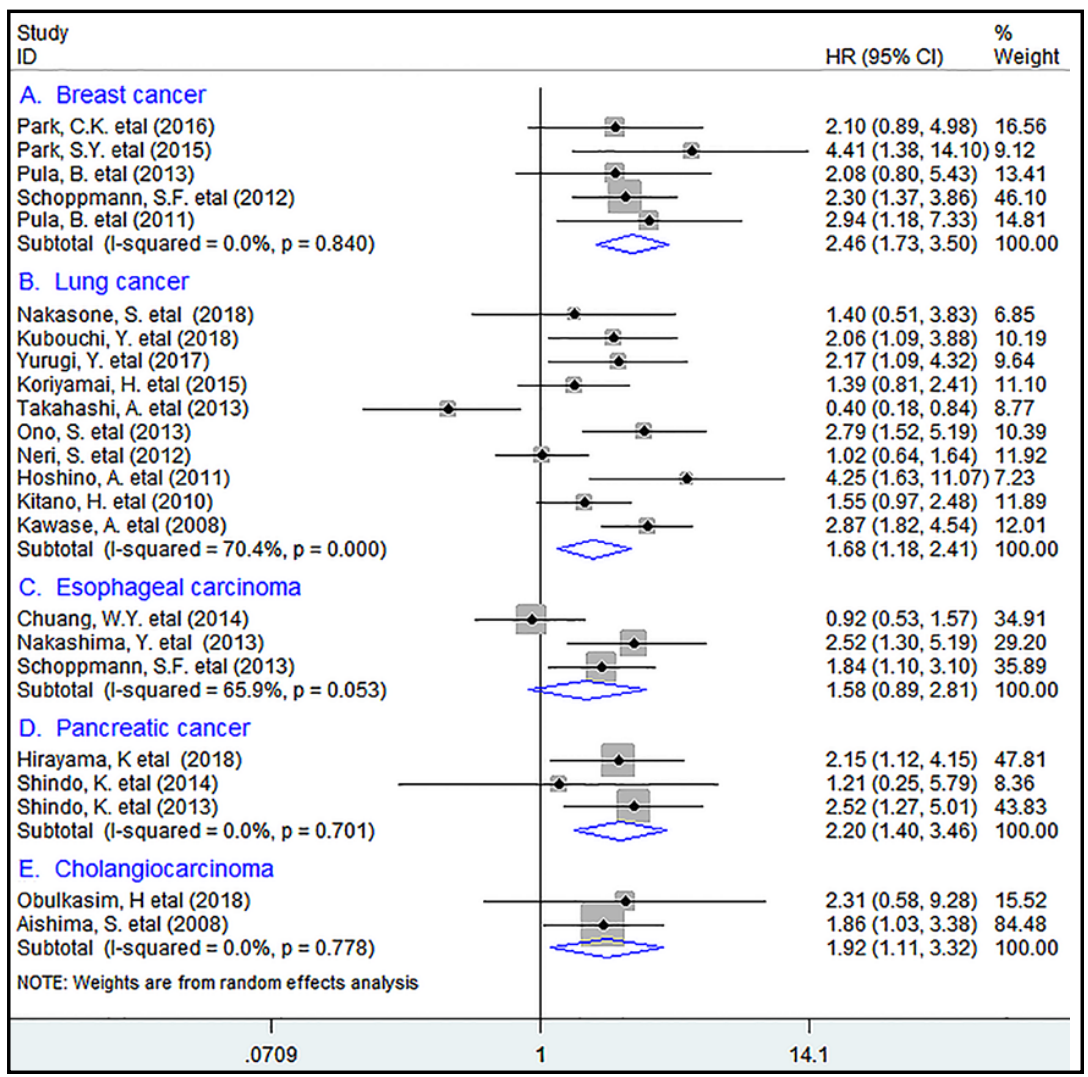

Fig. 4. Forest plot describing $\mathrm{HR}$ of the association between podoplanin $^{+} \quad$ fibroblast infiltration and DFS in solid tumors.

\begin{tabular}{|c|c|c|c|}
\hline Study & & & $\%$ \\
\hline ID & & $\mathrm{HR}(95 \% \mathrm{Cl})$ & Weight \\
\hline Cai, D.Y. etal (2017) & 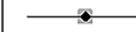 & $3.93(1.52,10.17)$ & 5.04 \\
\hline Park, C.K. etal (2016) & 2 & $3.46(1.41,8.48)$ & 5.29 \\
\hline Schoppmann, S.F. etal (2012) & 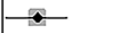 & $1.78(1.07,2.96)$ & 7.17 \\
\hline Pula, B. etal (2011) & 2 & $1.91(0.89,4.08)$ & 5.93 \\
\hline Nakasone, S. etal (2018) & $a$ & $1.76(0.88,3.56)$ & 6.23 \\
\hline Kubouchi, Y. etal (2018) & 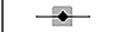 & $2.73(1.75,4.26)$ & 7.46 \\
\hline Yurugi, Y. etal (2017) & 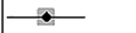 & $2.08(1.09,3.96)$ & 6.50 \\
\hline Takahashi, A. etal (2013) & & $0.38(0.19,0.75)$ & 6.29 \\
\hline Ono, S. etal (2013) & 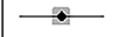 & $2.67(1.33,5.34)$ & 6.25 \\
\hline Ito, M. etal (2012) & $\infty$ & $3.47(1.13,10.65)$ & 4.34 \\
\hline Hoshino, A. etal (2011) & $\approx$ & $2.68(1.06,6.77)$ & 5.15 \\
\hline Schoppmann, S.F. etal (2013) & 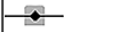 & $1.70(1.04,2.76)$ & 7.26 \\
\hline Hirayama, K etal (2018) & 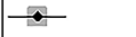 & $1.80(1.12,2.90)$ & 7.32 \\
\hline Shindo, K. etal (2013) & 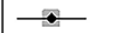 & $2.25(1.28,3.97)$ & 6.89 \\
\hline Choi, S.Y. etal (2013) & - & $0.73(0.41,1.30)$ & 6.84 \\
\hline Yamanashi, T. etal (2009) & & $0.13(0.04,0.42)$ & 4.07 \\
\hline Sun, W.Y. etal (2011) & & $8.33(1.05,66.35)$ & 1.97 \\
\hline Overall (I-squared $=74.2 \%, p=0.000$ ) & & $1.74(1.25,2.42)$ & 100.00 \\
\hline NOTE: Weights are from random effects analysis & & & \\
\hline $\begin{array}{cc} \\
0151\end{array}$ & $\begin{array}{l}1 \\
1\end{array}$ & 66.4 & \\
\hline
\end{tabular}

In addition, we found that increased density of these cells was significantly associated with clinicopathological features including lymph node metastasis $(\mathrm{OR}=1.47,95 \% \mathrm{CI} 1.12$ to $1.92, P=0.005)$, TNM stage (OR $=0.53,95 \% \mathrm{CI} 0.30$ to $0.94, P=0.030)$, lymphatic invasion $(\mathrm{OR}=1.93,95 \% \mathrm{CI} 1.13$ to $3.29, P=0.016)$ and vascular invasion $(\mathrm{OR}=3.00,95 \% \mathrm{CI} 1.34$ to $6.70, P=0.007)$, but not with primary tumor stage (OR $=1.32,95 \% \mathrm{CI} 0.46$ to $3.78, P=0.601$ ) or tumor differentiation $(\mathrm{OR}=0.54,95 \% \mathrm{CI} 0.25$ to $1.18, P=0.124)$ of patients. 


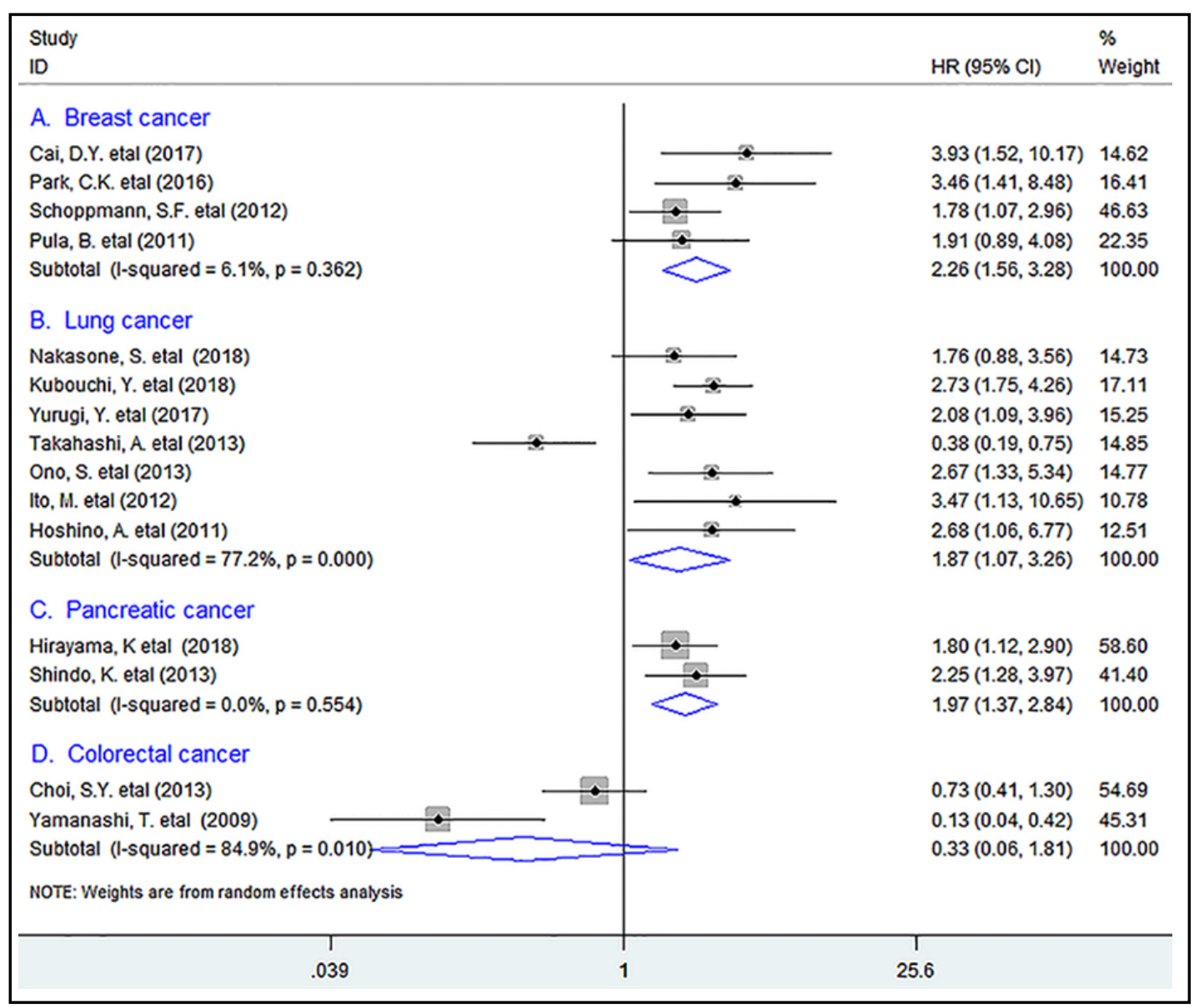

Fig. 5. Stratified analyses describing HRs of the association between podoplanin ${ }^{+}$fibroblast infiltration and DFS.

Sensitivity analysis. Sensitivity analysis showed that each included study had no influence on the overall HR for OS or DFS.

Publication bias. There was no publication bias between tumor-infiltrating podoplanin ${ }^{+}$ fibroblasts and OS $(P=0.965)$ or DFS $(P=0.387)$ in patients according to the Funnel plot and Egger's test.

\section{Discussion}

Fibroblasts are traditionally implicated and well recognized in wound healing and tissue fibrosis. In the past decades, although many studies have correlated tumor-infiltrating podoplanin ${ }^{+}$fibroblasts and prognosis of solid tumors, their results were not consistent even controversial. In the present meta-analysis, we found that podoplanin ${ }^{+}$fibroblast infiltration had a negative prognostic effect associated with survival in solid tumors especially in BC, LC, PC and CC. In addition, increased density of these cells was significantly associated with lymph node metastasis, TNM stage, lymphatic and vascular invasion of solid tumor. Hence, we think these findings provide meaningful statistical evidence to exhibit the negative prognostic role of these cells in human solid tumors.

The following reasons could possibly be responsible for the close association between increased tumor-infiltrating podoplanin ${ }^{+}$fibroblasts and worse outcome of patients presented in this study: Fibroblasts in the TME are able to promote tumor cell invasion, 
proliferation and survival through releasing growth factors, cytokines [39] and extracellular matrix-degrading proteases such as matrix metalloproteinases (MMPs) (e.g. MMP1) [40]. More importantly, podoplanin expressed in fibroblasts can enhance the ability of these cells to promote motility and survival of neighboring cancer cells [41]. Podoplanin ${ }^{+}$fibroblasts within tumor can secret angiogenic factors including IL-8 and TNF- $\alpha$ as well as VEGF which promote the formation of blood vessels thereby facilitating tumor growth [42]. In addition, they can also inhibit antitumor immunity via producing amount of immunosuppressive cytokines such as TGF- $\beta 1$ and IL-10 [42] and decrease the activation of effector immune cells such as CD8 ${ }^{+} \mathrm{T}$ cells through their acquisition of adhesion molecules [43] thereby establishing an immunosuppressive microenvironment. Hence, we can deduce that the tumor-infiltrating podoplanin ${ }^{+}$fibroblasts are able to promote tumor progression therefore decreasing survival.

There were some limitations in this study. First, morphometric analyses for podoplanin ${ }^{+}$ fibroblasts used in individual included studies were not consistent. In addition, studies with negative results may not be published, which can cause potential publication bias.

\section{Conclusion}

In conclusion, podoplanin ${ }^{+}$fibroblast infiltration leads to an unfavorable clinical outcome of patients with solid tumor, implicating that it is a valuable prognostic biomarker and targeting it may have a potential for effective treatment.

\section{Abbreviations}

OS (overall survival); DFS (disease-free survival); HR (hazard ratio); OR (odds ratios); $\mathrm{Cl}$ (confidence interval); NOS (Newcastle-Ottawa Scale); TNM (Tumor Lymph Node Metastasis); BC (breast cancer); LC (lung cancer); LAC (Lung adenocarcinoma); LSCC (lung squamous cell carcinoma); LNEC (Neuroendocrine carcinomas of the lung); PC (pancreatic cancer); CC (cholangiocarcinoma); EC (esophageal carcinoma); CRC (colorectal cancer); TPC (Thyroid papillary carcinoma); IHC (immunohistochemistry); TME (tumor microenvironment); NR (not reported); M (male); F (female); H (high); L (low).

\section{Acknowledgements}

GM. H. conceived of the study: participated in its design, extracted data, performed the statistical analysis and drafted the manuscript; SM. W., F. X. and KF. Z. participated in the statistical analysis; QN. D. participated in manuscript revision; W. C. participated in data extraction; Q. X. participated in its design and manuscript revision; LM.H. participated in its design and the statistical analysis. All authors read and approved the final manuscript.

This work was funded by the National Natural Science Foundation of China (Grant No. $81702803, \mathrm{GMH}$ ). This work was also granted from the project of Zhejiang Province Scientific Research Foundation of Traditional Chinese Medicine (Grant No. 2017ZB089, LMH) and Science and Technology Innovation Project of Shaoxing Health and Family Planning Program (Grant No. 2016CX002, WC).

\section{Disclosure Statement}

The authors declare no potential competing interests. 


\section{Cellular Physiology Cell Physiol Biochem 2018;51:1041-1050

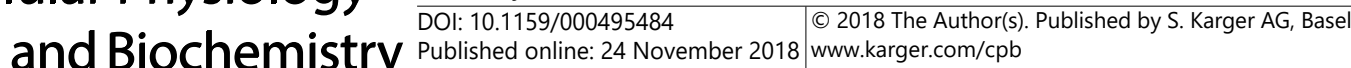

Hu et al.: Prognostic Role of Tumor-Infiltrating Podoplanin ${ }^{+}$Fibroblasts

\section{References}

1 Kalluri R: The biology and function of fibroblasts in cancer. Nature reviews Cancer 2016;16:582-598.

2 Wicki A, Christofori G: The potential role of podoplanin in tumour invasion. British journal of cancer 2007;96:1-5.

3 Astarita JL, Acton SE, Turley SJ: Podoplanin: emerging functions in development, the immune system, and cancer. Frontiers in immunology 2012;3:283.

4 Pula B, Witkiewicz W, Dziegiel P, Podhorska-Okolow M: Significance of podoplanin expression in cancerassociated fibroblasts: a comprehensive review. Int J Oncol 2013;42:1849-1857.

5 Stang A: Critical evaluation of the Newcastle-Ottawa scale for the assessment of the quality of nonrandomized studies in meta-analyses. European journal of epidemiology 2010;25:603-605.

6 Higgins JP, Thompson SG, Deeks JJ, Altman DG: Measuring inconsistency in meta-analyses. Bmj 2003;327:557-560.

7 Kuritz SJ, Landis JR, Koch GG: A general overview of Mantel-Haenszel methods: applications and recent developments. Annual review of public health 1988;9:123-160.

-8 DerSimonian R, Kacker R: Random-effects model for meta-analysis of clinical trials: an update. Contemporary clinical trials 2007;28:105-114.

-9 Egger M, Davey Smith G, Schneider M, Minder C: Bias in meta-analysis detected by a simple, graphical test. Bmj 1997;315:629-634.

10 Cai D, Wu X, Hong T, Mao Y, Ge X, Hua D: CD61+ and CAF+ were found to be good prognosis factors for invasive breast cancer patients. Pathol Res Pract 2017;213:1296-1301.

11 Park CK, Jung WH, Koo JS: Expression of cancer-associated fibroblast-related proteins differs between invasive lobular carcinoma and invasive ductal carcinoma. Breast cancer research and treatment 2016;159:55-69.

12 Park SY, Kim HM, Koo JS: Differential expression of cancer-associated fibroblast-related proteins according to molecular subtype and stromal histology in breast cancer. Breast cancer research and treatment 2015;149:727-741.

13 Pula B, Wojnar A, Witkiewicz W, Dziegiel P, Podhorska-Okolow M: Podoplanin expression in cancerassociated fibroblasts correlates with VEGF-C expression in cancer cells of invasive ductal breast carcinoma. Neoplasma 2013;60:516-524.

14 Schoppmann SF, Berghoff A, Dinhof C, Jakesz R, Gnant M, Dubsky P, Jesch B, Heinzl H, Birner P: Podoplaninexpressing cancer-associated fibroblasts are associated with poor prognosis in invasive breast cancer. Breast cancer research and treatment 2012;134:237-244.

15 Pula B, Jethon A, Piotrowska A, Gomulkiewicz A, Owczarek T, Calik J, Wojnar A, Witkiewicz W, Rys J, Ugorski M, Dziegiel P, Podhorska-Okolow M: Podoplanin expression by cancer-associated fibroblasts predicts poor outcome in invasive ductal breast carcinoma. Histopathology 2011;59:1249-1260.

-16 Nakasone S, Mimaki S, Ichikawa T, Aokage K, Miyoshi T, Sugano M, Kojima M, Fujii S, Kuwata T, Ochiai A, Tsuboi M, Goto K, Tsuchihara K, Ishii G: Podoplanin-positive cancer-associated fibroblast recruitment within cancer stroma is associated with a higher number of single nucleotide variants in cancer cells in lung adenocarcinoma. Journal of cancer research and clinical oncology 2018;144:893-900.

17 Kubouchi Y, Yurugi Y, Wakahara M, Sakabe T, Haruki T, Nosaka K, Miwa K, Araki K, Taniguchi Y, Shiomi T, Nakamura H, Umekita Y: Podoplanin expression in cancer-associated fibroblasts predicts unfavourable prognosis in patients with pathological stage IA lung adenocarcinoma. Histopathology 2018;72:490-499.

18 Yurugi Y, Wakahara M, Matsuoka Y, Sakabe T, Kubouchi Y, Haruki T, Nosaka K, Miwa K, Araki K, Taniguchi Y, Shiomi T, Nakamura H, Umekita Y: Podoplanin Expression in Cancer-associated Fibroblasts Predicts Poor Prognosis in Patients with Squamous Cell Carcinoma of the Lung. Anticancer Res 2017;37:207-213.

19 Koriyama H, Ishii G, Yoh K, Neri S, Morise M, Umemura S, Matsumoto S, Niho S, Ohmatsu H, Tsuboi M, Goto K, Ochiai A: Presence of podoplanin-positive cancer-associated fibroblasts in surgically resected primary lung adenocarcinoma predicts a shorter progression-free survival period in patients with recurrences who received platinum-based chemotherapy. Journal of cancer research and clinical oncology 2015;141:11631170. 


\section{Cellular Physiology Cell Physiol Biochem 2018;51:1041-1050 and Biochemistry DOl: 10.1159/000495484 2018 O 2018 The Author(s). Published by S. Karger AG, Basel

Hu et al.: Prognostic Role of Tumor-Infiltrating Podoplanin ${ }^{+}$Fibroblasts

20 Takahashi A, Ishii G, Kinoshita T, Yoshida T, Umemura S, Hishida T, Yoh K, Niho S, Goto K, Ohmatsu H, Ohe Y, Nagai K, Ochiai A: Identification of prognostic immunophenotypic features in cancer stromal cells of high-grade neuroendocrine carcinomas of the lung. Journal of cancer research and clinical oncology 2013;139:1869-1878.

21 Ono S, Ishii G, Nagai K, Takuwa T, Yoshida J, Nishimura M, Hishida T, Aokage K, Fujii S, Ikeda N, Ochiai A: Podoplanin-positive cancer-associated fibroblasts could have prognostic value independent of cancer cell phenotype in stage I lung squamous cell carcinoma: usefulness of combining analysis of both cancer cell phenotype and cancer-associated fibroblast phenotype. Chest 2013;143:963-970.

-22 Neri S, Ishii G, Taira T, Hishida T, Yoshida J, Nishimura M, Nagai K, Ochiai A: Recruitment of podoplanin positive cancer-associated fibroblasts in metastatic lymph nodes predicts poor prognosis in pathological N2 stage III lung adenocarcinoma. Ann Surg Oncol 2012;19:3953-3962.

23 Ito M, Ishii G, Nagai K, Maeda R, Nakano Y, Ochiai A: Prognostic impact of cancer-associated stromal cells in patients with stage I lung adenocarcinoma. Chest 2012;142:151-158.

24 Hoshino A, Ishii G, Ito T, Aoyagi K, Ohtaki Y, Nagai K, Sasaki H, Ochiai A: Podoplanin-positive fibroblasts enhance lung adenocarcinoma tumor formation: podoplanin in fibroblast functions for tumor progression. Cancer Res 2011;71:4769-4779.

25 Kitano H, Kageyama S, Hewitt SM, Hayashi R, Doki Y, Ozaki Y, Fujino S, Takikita M, Kubo H, Fukuoka J: Podoplanin expression in cancerous stroma induces lymphangiogenesis and predicts lymphatic spread and patient survival. Archives of pathology \& laboratory medicine 2010;134:1520-1527.

-26 Kawase A, Ishii G, Nagai K, Ito T, Nagano T, Murata Y, Hishida T, Nishimura M, Yoshida J, Suzuki K, Ochiai A: Podoplanin expression by cancer associated fibroblasts predicts poor prognosis of lung adenocarcinoma. Int J Cancer 2008;123:1053-1059.

27 Chuang WY, Yeh CJ, Chao YK, Liu YH, Chang YS, Tseng CK, Chang HK, Wan YL, Hsueh C: Concordant podoplanin expression in cancer-associated fibroblasts and tumor cells is an adverse prognostic factor in esophageal squamous cell carcinoma. International journal of clinical and experimental pathology 2014;7:4847-4856.

-28 Nakashima Y, Yoshinaga K, Kitao H, Ando K, Kimura Y, Saeki H, Oki E, Morita M, Kakeji Y, Hirahashi M, Oda Y, Maehara Y: Podoplanin is expressed at the invasive front of esophageal squamous cell carcinomas and is involved in collective cell invasion. Cancer science 2013;104:1718-1725.

29 Schoppmann SF, Jesch B, Riegler MF, Maroske F, Schwameis K, Jomrich G, Birner P: Podoplanin expressing cancer associated fibroblasts are associated with unfavourable prognosis in adenocarcinoma of the esophagus. Clinical \& experimental metastasis 2013;30:441-446.

-30 Hirayama K, Kono H, Nakata Y, Akazawa Y, Wakana H, Fukushima H, Fujii H: Expression of podoplanin in stromal fibroblasts plays a pivotal role in the prognosis of patients with pancreatic cancer. Surgery today 2018;48:110-118.

-31 Shindo K, Aishima S, Ohuchida K, Fujino M, Mizuuchi Y, Hattori M, Ohtsuka T, Tokunaga S, Mizumoto K, Tanaka M, Oda Y: Podoplanin expression in the cyst wall correlates with the progression of intraductal papillary mucinous neoplasm. Virchows Archiv : an international journal of pathology 2014;465:265-273.

-32 Shindo K, Aishima S, Ohuchida K, Fujiwara K, Fujino M, Mizuuchi Y, Hattori M, Mizumoto K, Tanaka M, Oda Y: Podoplanin expression in cancer-associated fibroblasts enhances tumor progression of invasive ductal carcinoma of the pancreas. Molecular cancer 2013;12:168.

-33 Choi SY, Sung R, Lee SJ, Lee TG, Kim N, Yoon SM, Lee EJ, Chae HB, Youn SJ, Park SM: Podoplanin, alphasmooth muscle actin or S100A4 expressing cancer-associated fibroblasts are associated with different prognosis in colorectal cancers. Journal of Korean medical science 2013;28:1293-1301.

-34 Yamanashi T, Nakanishi Y, Fujii G, Akishima-Fukasawa Y, Moriya Y, Kanai Y, Watanabe M, Hirohashi S: Podoplanin expression identified in stromal fibroblasts as a favorable prognostic marker in patients with colorectal carcinoma. Oncology 2009;77:53-62.

-35 Obulkasim H, Shi X, Wang J, Li J, Dai B, Wu P, Wang S, Wang X, Ding Y: Podoplanin is an important stromal prognostic marker in perihilar cholangiocarcinoma. Oncology letters 2018;15:137-146.

-36 Aishima S, Nishihara Y, Iguchi T, Taguchi K, Taketomi A, Maehara Y, Tsuneyoshi M: Lymphatic spread is related to VEGF-C expression and D2-40-positive myofibroblasts in intrahepatic cholangiocarcinoma. Modern pathology : an official journal of the United States and Canadian Academy of Pathology, Inc 2008;21:256-264. 


\section{Cellular Physiology Cell Physiol Biochem 2018;51:1041-1050 \begin{tabular}{l|l|l} 
DOl: 10.1159/000495484 & (O 2018 The Author(s). Published by S. Karger AG, Basel
\end{tabular}

-37 Kan S, Konishi E, Arita T, Ikemoto C, Takenaka H, Yanagisawa A, Katoh N, Asai J: Podoplanin expression in cancer-associated fibroblasts predicts aggressive behavior in melanoma. Journal of cutaneous pathology 2014;41:561-567.

-38 Sun WY, Jung WH, Koo JS: Expression of cancer-associated fibroblast-related proteins in thyroid papillary carcinoma. Tumour Biol 2016;37:8197-8207.

39 Bruzzese F, Hagglof C, Leone A, Sjoberg E, Roca MS, Kiflemariam S, Sjoblom T, Hammarsten P, Egevad L, Bergh A, Ostman A, Budillon A, Augsten M: Local and systemic protumorigenic effects of cancer-associated fibroblast-derived GDF15. Cancer Res 2014;74:3408-3417.

-40 Boire A, Covic L, Agarwal A, Jacques S, Sherifi S, Kuliopulos A: PAR1 is a matrix metalloprotease-1 receptor that promotes invasion and tumorigenesis of breast cancer cells. Cell 2005;120:303-313.

-41 Ito S, Ishii G, Hoshino A, Hashimoto H, Neri S, Kuwata T, Higashi M, Nagai K, Ochiai A: Tumor promoting effect of podoplanin-positive fibroblasts is mediated by enhanced RhoA activity. Biochem Biophys Res Commun 2012;422:194-199.

42 Poggi A, Musso A, Dapino I, Zocchi MR: Mechanisms of tumor escape from immune system: role of mesenchymal stromal cells. Immunology letters 2014;159:55-72.

43 Powell DW: Myofibroblasts: paracrine cells important in health and disease. Transactions of the American Clinical and Climatological Association 2000;111:271-292; discussion 292-273. 